\title{
Upper respiratory tract infection, heterologous immunisation and meningococcal disease
}

\author{
R. J. P. M. SCHOLTEN*, H. A. BIJLMER†, H. TOB $\ddagger, J$. DANKERT§ and L. M. BOUTER* $\ddagger$ \\ * Institute for Research in Extramural Medicine, Vrije Universiteit, Amsterdam, †Hospital 'Bronovo', The \\ Hague, $\$$ Department of Epidemiology and Biostatistics, Vrije Universiteit, Amsterdam and $\S$ Netherlands \\ Reference Laboratory for Bacterial Meningitis, WHO Collaborating Centre, University of Amsterdam/National \\ Institute for Public Health and Environmental Protection, Amsterdam. The Netherlands
}

To test the hypothesis that an episode of upper respiratory tract infection or heterologous immunisation is a predisposing factor for the occurrence of meningococcal disease, data from 377 cases of meningococcal disease and their household contacts $(n=1124)$ were analysed by conditional logistic regression analysis with stratification for household. The odds ratio for a recent upper respiratory tract infection for patients versus household contacts, adjusted for age and the presence of an underlying predisposing disease, was 2.8 and that for recent heterologous immunisation 1.0. These results support previous observations regarding the association between a preceding upper respiratory tract infection and the occurrence of meningococcal disease; however, no association was found between preceding heterologous immunisation and meningococcal disease. Therefore, increased alertness after heterologous immunisation does not seem warranted.

\section{Introduction}

Meningococcal disease still poses major health problems in both developing and industrialised countries. The high case-fatality rates and significant sequelae resulting from this disease continue to be of considerable concern. Notwithstanding the scientific progress that has been made in knowledge of Neisseria meningitidis, the reason why the majority of individuals who acquire meningococci become carriers, and only a minority develop a life-threatening disease, remains obscure.

Several factors have been recognised as risk factors for the occurrence of meningococcal disease. These factors include young age (due to low titres of bactericidal antibodies), male gender, diseases that compromise the immunological defence mechanisms (including complement deficiencies), low socio-economic status, crowding, passive smoking and environmental factors [1-4]. Many reports have demonstrated that a preceding upper respiratory tract infection due to influenza $\mathrm{A}$,

Received 19 Aug. 1998; accepted 15 Feb. 1999.

Corresponding author: Dr R. J. P. M. Scholten (e-mail: rjpm.scholten.emgo@med.vu.nl). other viruses or Mycoplasma pneumoniae is a predisposing factor for the occurrence of meningococcal disease [5-8]. Heterologous immunisation may be associated with the occurrence of invasive bacterial disease (including meningococcal disease); however, reports on this subject have been conflicting [9-13].

During a nationwide survey in the Netherlands from 1989 to 1990, data were collected from cases of meningococcal disease and their household contacts [14]. This data set made it possible to analyse the association between recent upper respiratory tract infection and heterologous immunisation, and the occurrence of meningococcal disease.

\section{Patients and methods}

This study included all consecutive patients with meningococcal disease, from whom the causative meningococcus or other diagnostic material had been forwarded to the Netherlands Reference Laboratory for Bacterial Meningitis in Amsterdam between 1 April 1989 and 30 April 1990. For logistic reasons the study was interrupted during the summer months from July to September 1989.

During the study period 509 patients with meninogo- 
coccal disease were reported. Two co-primary cases, who were admitted to hospital within a few hours after the primary patient, and five secondary cases, who were admitted 2-35 days after the primary patient, were excluded from the analysis [14], leaving 502 eligible cases of meningococcal disease. In 471 patients, $N$. meningitidis was isolated from the cerebrospinal fluid (CSF) or blood or both, in nine patients the diagnosis was based on the presence of meningococcal capsular polysaccharide antigens in the CSF and in 22 patients there was a clinical syndrome of meningitis or septicaemia with fever and a petechial rash, considered to be meningococcal disease by the attending physician.

Persons who slept in the same house as the patient during the week prior to hospital admission of the patient were considered as household contacts. Every patient and household was visited by a regional Public Health Officer as soon as possible after hospital admission of the patient. Socio-demographic data (including the composition of the household) and information concerning the presence of predisposing diseases, recent use of antibiotics, a recent episode of upper respiratory tract infection and recent immunisation were collected by means of a questionnaire. The questionnaires pertaining to subjects of young age were filled out by a parent or a guardian of the subject.

Predisposing diseases for meningococcal disease were defined as the presence of malignancy, immunosuppressive therapy, diabetes mellitus, chronic obstructive lung disease, chronic renal failure, liver cirrhosis, intravenous drug abuse, recent severe head injury and a history of neurosurgery.

A recent episode of upper respiratory tract infection was defined as the occurrence of symptoms of a common cold, a sore throat, 'flu', ear infection or bronchitis in the 4 weeks prior to hospital admission of the patient.

A subject was considered to be recently immunised if one of the following vaccines had been administered in the 4 weeks prior to hospital admission: diphtheria toxoid, polio, tetanus toxoid and pertussis vaccine (DPTP); measles, mumps and rubella vaccine (MMR); tetanus toxoid, typhoid, hepatitis B or influenza vaccine.

Analysis was done by multiple conditional logistic regression with stratification for household. The following potential confounders were addressed: age, gender, the presence of a predisposing disease and use of antibiotics during the 4 weeks prior to hospital admission of the patient. To enhance statistical efficiency, age was classified post hoc into seven functional categories which represented homogeneous age groups with respect to the occurrence of meningococcal disease. The age categories were $<1$ year, 1 year, 2-4, 5-14, 15-19, 20-49 and $\geqslant 50$ years, respectively. Indicator variables were constructed for each age category and the category $20-49$ years served as the reference category. The effect of recent immunisation was analysed in two different logistic models. In the first model, an indicator term for recent immunisation with any of the above-mentioned vaccines was used (model 1). In the second model, two indicator terms were constructed, the first indicator pertaining to recent immunisation with a vaccine that included the measles component (MMR) and the second indicator referring to all other types of vaccines.

All analyses were performed with the statistical packages SPSS $^{\mathrm{R}}$ and EGRET ${ }^{\mathrm{R}}$.

\section{Results}

Of the 502 eligible patients, 19 were living alone. Data were obtained from 377 of the remaining 483 patients (response rate $78.1 \%$ ) and their household contacts $(\mathrm{n}=1124)$. The median number of household contacts per patient was 3 (range 1-15).

The distribution of the various potential confounders and study variables among cases and household contacts is shown in Table 1. The distribution of gender was similar in both groups. The age distribution of patients and household contacts differed substantially, patients being younger on the average. Cases were suffering more often from a predisposing disease and had used antibiotics prior to hospital admission slightly more often than the household contacts.

During the 4 weeks prior to admission of the patient to hospital, an upper respiratory tract infection had been present in 134 of the patients $(35.5 \%)$ and in 216 of the household contacts $(19.2 \%)$, and heterologous immunisation in $36(9.5 \%)$ and $29(2.6 \%)$, respectively (Table 1). The type of vaccine which had been administered is shown in Table 2.

The inclusion of gender and recent use of antibiotics into the logistic models did not change the estimated coefficients of the study variables substantially and were left out of the model. In both logistic models, the odds ratio for a recent upper respiratory tract infection of patients versus household contacts was 2.8 (95\% confidence interval [95\% CI] 1.8-4.2), adjusted for age and the presence of a predisposing disease in the conditional logistic regression analysis, with stratification for household. In model 1 , the odds ratio for recent immunisation with any vaccine was $1.0(95 \%$ CI: 0.5-2.2). When separate indicator terms for recent immunisation with MMR and other vaccines were used (model 2), the odds ratio for recent immunisation with MMR was $0.8(95 \% \mathrm{CI}: 0.2-2.9)$ and that for any other type of vaccine was 1.1 (95\% CI: $0.5-2.7)$. 
Table 1. Distribution of potential confounders and study variables among 377 cases of meningococcal disease and their household contacts $(\mathrm{n}=1124)$

\begin{tabular}{|c|c|c|c|c|}
\hline \multirow[b]{2}{*}{ Variable } & \multicolumn{4}{|c|}{ Number of subjects/total number of subjects (\%) } \\
\hline & \multicolumn{2}{|c|}{ Patients } & \multicolumn{2}{|c|}{ Household contacts } \\
\hline \multicolumn{5}{|l|}{$\begin{array}{l}\text { Potential confounders } \\
\text { Age category (years) }\end{array}$} \\
\hline$<1$ & $38 / 377$ & $(10.1)$ & $19 / 1124$ & (1.7) \\
\hline 1 & $40 / 377$ & $(10.6)$ & $12 / 1124$ & (1.1) \\
\hline $2-4$ & $79 / 377$ & $(21.0)$ & $81 / 1124$ & (7.2) \\
\hline $5-14$ & $112 / 377$ & $(29.7)$ & $202 / 1124$ & $(18.0)$ \\
\hline $15-19$ & $60 / 377$ & (15.9) & $106 / 1124$ & $(9.4)$ \\
\hline $20-49$ & $32 / 377$ & $(8.5)$ & $651 / 1124$ & (57.9) \\
\hline$\geqslant 50$ & $16 / 377$ & (4.2) & $53 / 1124$ & (4.7) \\
\hline Male gender & $197 / 377$ & $(52.3)$ & $584 / 1124$ & (52.0) \\
\hline Predisposing disease present & $20 / 374$ & $(5.3)$ & $36 / 1121$ & (3.2) \\
\hline Recent use of antibiotics* & $16 / 350$ & $(4.6)$ & $41 / 1121$ & (3.7) \\
\hline \multicolumn{5}{|l|}{ Study variables } \\
\hline Recent upper respiratory tract infection* & $134 / 377$ & $(35.5)$ & $216 / 1124$ & $(19.2)$ \\
\hline Recent heterologous immunisation* & $36 / 377$ & $(9.5)$ & $29 / 1119$ & $(2.6)$ \\
\hline
\end{tabular}

${ }^{*}$ During the 4 weeks prior to hospital admission of the patient.

Table 2. Type of vaccine administered to cases of meningococcal disease and household contacts during the 4 weeks before hospital admission of the patient

\begin{tabular}{lcc}
\hline & \multicolumn{2}{c}{ Number of recipients } \\
\cline { 2 - 3 } Vaccine & $\begin{array}{c}\text { Patients } \\
(\mathrm{n}=36)\end{array}$ & $\begin{array}{c}\text { Households contacts } \\
(\mathrm{n}=29)\end{array}$ \\
\hline DPTP & 22 & 15 \\
MMR & 11 & 2 \\
DPTP and MMR & 2 & 7 \\
Tetanus toxoid & 0 & 1 \\
Typhoid & 1 & 0 \\
Hepatitis B & 0 & 1 \\
Influenza & 0 & 2 \\
Not indicated & 0 & 1 \\
\hline
\end{tabular}

\section{Discussion}

This report addresses the association of recent upper respiratory tract infection or immunisation, and the occurrence of meningococcal disease. The analysis was done by comparing cases of meningococcal disease and their household contacts in a matched analysis with stratification for household. In this way, cases and household contacts were matched on a number of important covariates, such as socio-economic status, crowding, environmental factors, genetic factors, passive smoking and season. The presence of a predisposing disease and age were important confounders which had to be adjusted for in the analysis. As expected, the age distributions of the two groups differed substantially; however, there was sufficient overlap of the age distributions of patients and household contacts to enable the adjustment for age in the multivariate analysis.

The response rate of the households during the survey was $78.1 \%$. It is not likely that non-response led to differential reporting of the events under study. Another possible limitation of the study is recall bias. It is possible that individuals who had been hospitalised recently because of a severe disease might be more likely to recall events in their lives preceding that disease than healthy contacts. However, this is unlikely to pertain to the recall of recent immunisation, because this procedure will have a significant impact on a subject's perception. Information bias might be present regarding the recall of a recent upper respiratory tract infection. However, it is likely that cases and their household contacts would have a more similar attitude to, and recall of, the events under study than cases and controls who were selected from different populations (and who were not family members). In addition, in many instances the parent or a guardian of a subject of young age (who constituted the majority of the cases), filled out the questionnaire. In those circumstances it is unlikely that there would be a different recall of a recent upper respiratory tract infection. If recall bias was a problem in the present study, the odds ratio for a recent upper respiratory tract infection of patients with meningococcal disease versus household contacts might be overestimated.

The results of the present study support previous observations regarding the association between an upper respiratory tract infection and the occurrence of meningococcal disease [5-8]. This predisposing effect has been explained by several mechanisms, including damage to the mucosal surfaces by the respiratory pathogen facilitating the invasion of the meningococcus into the bloodstream, and a temporary suppression of the immune system due to the viral infection. The latter mechanism may also play a role in the relationship between meningococcal disease and preceding heterologous immunisation, although no significant association was found. There was only a small number of recently immunised subjects in this study (36 patients and 29 household contacts), and lack of power might be one reason for not finding an association. However, in both logistic models the point estimates of the odds ratios of recent immunisation were near to the neutral 
value of 1 . Thus, if this study population is considered to be representative, the presence of a real effect was not very likely. The finding of a 'protective' odds ratio of 0.8 for recent immunisation with MMR in model 2 (although not statistically significant) was unusual and does not seem plausible. Indeed the measles component of MMR especially is thought to cause immunosuppression [15-16]. However, it should be borne in mind that the statistical methods that have been applied are based on large sample theory. As these calculations were based on only 13 patients and nine household contacts with recent MMR immunisation, the point estimate may be unreliable and the 'protective' odds ratio should be interpreted with caution. Studies with a larger number of recently immunised subjects are needed to refute or verify these findings.

The results regarding the effect of recent immunisation are in accordance with the findings of three other casecontrol studies [11-13]. However, in a double-blind placebo-controlled efficacy study of two acellular pertussis vaccines, four vaccinated children died during follow-up as a result of invasive bacterial disease. The authors had no plausible explanation for this finding [10]. They analysed a subset of the study population but could not find any indication of immunosuppression in vaccine recipients. Finally, in an observational study of 82 children, more infectious episodes occurred during the month following DTP immunisation compared with the month before immunisation [9]. No control group was included in this study and the influence of potential confounding factors cannot be excluded.

It is concluded that heterologous immunisation does not seem to be related to the occurrence of meningococcal disease, but that an upper respiratory tract infection enhances the risk of meningococcal disease. The exact mechanism by which this occurs cannot be deduced from this study. Damage to the mucosal surfaces, as postulated before by others, seems to be a likely explanation for this increased risk [5-8]. Increased alertness after heterologous immunisation may not be warranted.

We are most grateful to all Public Health Officers for their kind cooperation. We thank all medical microbiologists in the Netherlands for submitting bacterial isolates to the Netherlands Reference Laboratory for Bacterial Meningitis, and all specialists for their assistance in obtaining informed consent of the patients and their household contacts. This work was partially supported by a grant from the Praeventiefonds (grant no. 28-1874).

\section{References}

1. Goldschneider I, Gotschlich EC, Artenstein MS. Human immunity to the meningococcus. II. Development of natural immunity. $J$ Exp Med 1969; 129: 1327-1348.

2. Abbott JD, Jones DM, Painter MJ, Young SEJ. The epidemiology of meningococcal infections in England and Wales 1912-1983. J Infect 1985; 11: 241-257.

3. Fijen CAP, Kuijper EJ, Hannema AJ, Sjöholm AG, van Putten JPM. Complement deficiencies in patients over ten years old with meningococcal disease due to uncommon serogroups. Lancet 1989; 2: 585-588.

4. Stanwell-Smith RE, Stuart JM, Hughes AO, Robinson P, Griffin $\mathrm{MB}$, Cartwright $\mathrm{K}$. Smoking, the environment and meningococcal disease: a case control study. Epidemiol Infect 1994; 112: $315-328$.

5. Krasinski K, Nelson JD, Butler S, Luby JP, Kusmiesz H. Possible association of mycoplasma and viral respiratory infections with bacterial meningitis. Am J Epidemiol 1987; 125: 499-508.

6. Moore PS, Hierholzer J, DeWitt W et al. Respiratory viruses and Mycoplasma as cofactors for epidemic group A meningococcal meningitis. JAMA 1990; 264: 1271-1275.

7. Cartwright KAV, Jones DM, Smith AJ, Stuart JM, Kaczmarski EB, Palmer SR. Influenza A and meningococcal disease. Lancet 1991; 338: 554-557.

8. Hubert B, Watier L, Garnerin P, Richardson S. Meningococcal disease and influenza-like syndrome: a new approach to an old question. $J$ Infect Dis 1992; 166: 542-545.

9. Jaber L, Shohat M, Mimouni M. Infectious episodes following diphtheria-pertussis-tetanus vaccination: a preliminary observation in infants. Clin Pediatr 1988; 27: 491-494.

10. Storsaeter J, Olin P, Renemar B et al. Mortality and morbidity from invasive bacterial infections during a clinical trial of acellular pertussis vaccines in Sweden. Pediatr Infect Dis $J$ 1988; 7: 637-645

11. Black SB, Cherry JD, Shinefield HR, Fireman B, Christenson P, Lampert D. Apparent decreased risk of invasive bacterial disease after heterologous childhood immunization. Am J Dis Child 1991; 145: 746-749.

12. Davidson M, Letson GW, Ward JI et al. DTP immunization and susceptibility to infectious diseases. Is there a relationship? Am J Dis Child 1991; 145: 750-754.

13. Joffe LS, Glode MP, Gutierrez MK, Wiesenthal A, Luckey DW, Harken L. Diphtheria-tetanus toxoids-pertussis vaccination does not increase the risk of hospitalization with an infectious illness. Pediatr Infect Dis $J$ 1992; 11: 730-735.

14. Scholten RJPM, Bijlmer HA, Dankert J, Valkenburg HA. [Secondary cases of meningococcal disease in The Netherlands, 1989-1990; a reappraisal of chemoprophylaxis.] $\mathrm{Ned}$ Tijdschr Geneeskd 1993; 137: 1505-1508.

15. Griffin DE, Ward BJ, Esolen LM. Pathogenesis of measles virus infection: an hypothesis for altered immune responses. $J$ Infect Dis 1994; 170 Suppl 1: S24-S31.

16. Hussey GD, Goddard EA, Hughes $\mathrm{J}$ et al. The effect of Edmonston-Zagreb and Schwarz measles vaccines on immune responses in infants. $J$ Infect Dis 1996; 173: 1320-1326. 